September 21-25, 2020, Virtual, Online

GT2020-16173

\title{
ANALYSIS OF IGNITION PROCESSES AT COMBUSTORS FOR AERO ENGINES AT HIGH ALTITUDE CONDITIONS WITH AND WITHOUT EFFUSION COOLING
}

\author{
Alexios-Dionysios Martinos, Nikolaos Zarzalis, Stefan-Raphael Harth
}

Engler-Bunte-Institute, Karlsruhe Institute of Technology, Karlsruhe, Germany

\section{ABSTRACT}

The ability to re-ignite at high altitude after a flameout event is critical for flight safety. One reason that makes the relight process of the engine difficult is the low temperature and pressure, which leads to poor atomization, low degree of evaporation and slow reaction rate of the vaporized fuel. For this research work a rectangular, one sector $R Q L$ combustion chamber was utilized for experimental investigations at high altitude conditions. The design of the chamber is modular so that experiments for two configurations, i.e. without and with effusion cooling holes can be conducted. The fuel injection and the ignition system are representative of the ones used in commercial aviation.

The investigations were performed in the frame of the European research project SOPRANO at the ISCAR rig. The ISCAR rig is capable of generating low pressure and temperature conditions for flowing kerosene-air mixtures.

The investigation focuses on the characterization of the ignition process, in terms of probability, minimum fuel to air ratio (FAR) and ignition timing for a successful ignition event. In addition, the unsteady flame kernel generation and propagation were analyzed by high-speed imaging recording.

An in-house image processing code was developed in order to derive quantitative spatial information of the flame and overall trends among ignition sequences for the same or different operating conditions.

In order to achieve comparability between the investigated configurations (liners without and with effusion cooling), the pressure drop across the nozzle and the liners was the same depending on the operating condition. Results show that both pressure and temperature affect the ignition process, with the former being the dominant parameter in the investigated conditions. In both configurations, the minimum FAR increased as long as the conditions in the chamber became more adverse, indicating that at high altitude low-pressure situations, the performance of the airblast atomizer deteriorated causing poor ignition. This is overcome by creating a richer fuel-air mixture in the primary zone. Finally, the air injected through the effusion cooling holes near the spark seems to create favorable conditions for the ignition process.

\section{NOMENCLATURE}

$\mathrm{D}_{\mathrm{h}} \quad$ Hydraulic mean diameter

$\mathrm{D}_{\mathrm{p}} \quad$ Diameter of the prefilmer

$\mathrm{H}_{0} \quad$ Height of the interrogation window

$\mathrm{L}_{0} \quad$ Length of the interrogation window

$\mathrm{P}_{3} \quad$ Pressure in the chamber

$\mathrm{T}_{3} \quad$ Temperature in the chamber

$\mu \quad$ Dynamic viscosity

$\rho \quad$ Density

$\sigma \quad$ Surface tension

$\Phi \quad$ Equivalence ratio

\section{Abbreviations:}

ALR Air to Liquid Ratio

FAR Fuel to Air Ratio

IRZ Inner Recirculation Zone

ISCAR Ignition under Sub atmospheric Conditions - Altitude Relight rig

KIT Karlsruhe Institute of Technology

ORZ Outer Recirculation Zone

RQL Rich Quench Lean

SMD Sauter Mean Diameter

SOPRANO SOot Processes and Radiation in Aeronautical inNOvative combustors 


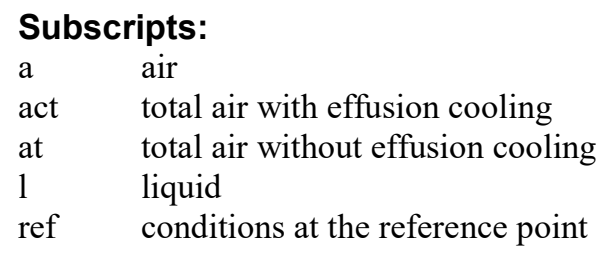

\section{INTRODUCTION}

The design of modern combustors has been challenging for engineers, due to the conflict of interest among emission standards, flame stability and the engine's relight. Future commercial aviation trends include concepts with reduced size of the combustor and lean burning to counter the harmful emissions such as nitrous oxides $\left(\mathrm{NO}_{\mathrm{x}}\right)$ and carbon monoxide (CO). Both designs could have adverse effects on the combustor's performance during a high altitude relight. The altitude relight capability of an aircraft engine is one of the requirements needed for the successful certification of an engine, due to the inherent safety implications [1]. Thus, it is a very important area of research. Altitude relight is a quite complex process influenced by both physical and chemical phenomena, such as chemical kinetics, fluid mechanics, atomization and fuel evaporation. The complexity of the phenomena governing the ignition process affects the reliability of the prediction of its behavior. A description of the chain of events during the engine's high altitude relight based on Mosbach et al. [3], can be given as follows.

Kerosene Jet-A1 is injected into the chamber and the spark igniter deposits energy at a rate of few $\mathrm{Hz}$, producing plasma spheres, which are transported by the incoming mixture in certain flow directions dependent on the instantaneous conditions of the highly turbulent flow field. The order of magnitude of the initial temperature of the plasma is several thousand degrees but decreases rapidly. Reactions are then initiated and the ignition kernel has been generated. The formation of a stable flame depends strongly on the subsequent development of the flame kernel. In this sequence of events, several parameters have influence and probably the most important are the flow field, local fuel to air ratio, droplet sizes, and ignition energy. There is a number of investigations on the ignition of spray flames, which have been reported in the literature, evaluating the influence of various parameters on the ignition process under well defined experimental conditions [2]. However, experiments under realistic conditions of an altitude relight are scarce [3-5].

Full-scale engine tests under relight conditions are challenging on a technical and economical perspective and the possibilities for instrumentation are usually limited. The simulation tools for two-phase combustible mixtures are computationally expensive, still under development and need experimental validation. Non-expensive models which predict the flame propagation following the spark ignition are valuable in assisting engineers during the early design of the combustors [6]. For this investigation, a one-sector combustion chamber with broad optical access was used for experimental investigations at high altitude relight conditions.

This research activity aims to determine the ignition capability of a realistic configuration under high altitude conditions and to improve the understanding/knowledge of the relevant phenomena.

\section{TEST RIG DESCRIPTION, TEST PROCEDURE}

The experiments of this research activity were conducted at the ISCAR ("Ignition under Sub atmospheric ConditionsAltitude Relight") rig, which was designed and manufactured at the Engler-Bunte-Institute of Karlsruhe Institute of Technology (KIT). It is capable of generating low pressure and temperature conditions for flowing kerosene-air mixtures. The influence of turbulence on spark ignition was studied previously at the ISCAR rig by Majcherczyk et al. [7]. The simplified picture of the rig is shown in Figure 1 and the corresponding schematic diagram in Figure 2.

The flow and low pressure in the ignition chamber are produced by a set of eight parallel ejectors, which are vacuum devices and can be switched on and off independently. The pressure can be regulated through pressure reducer 3 (PR3) in Figure 2. The cold air is generated through the expansion of compressed air in two small automotive turbochargers. The temperature at the exit of the turbine depends on the turbine pressure ratio and can be regulated by inlet turbine pressure through pressure reducer 1 (PR1) in Figure 2. The excess of cold air is used to cool down the walls of the incoming flow. This additional cooling allows maintaining a constant ignition chamber temperature even at low volume flows and at a lower temperature range. The practical operating temperature and pressure range of the rig is $-20^{\circ} \mathrm{C}$ to ambient temperature and 0.4 bar to atmospheric respectively.

The air mass flow in the ignition chamber is regulated through regulating valve 1 (RV1), as it is shown in Figure 2. The linear dependency of the mass flux from the suction pressure enforces a further regulation through a bypass system, through pressure reducer 2 and regulating valve 2 (PR2), (RV2). Thus, mass flux and pressure in the ignition chamber can be regulated independently of the linear ejector characteristic curve.

The fuel (kerosene Jet-A1) is supplied from a pressure vessel, pushed out by a piston. The temperature of the kerosene- Jet-A1 is expected to be a few degrees lower than the room temperature, due to the exposure of the fuel pipes in the cold air during the experimental trial.

The combustion chamber shown in Figure 3 was developed by KIT within the scope of the SOPRANO EU project. It is a single injection RQL combustion chamber suitable to operate under high altitude conditions. Dilution and effusion cooling holes have been sized to reproduce the realistic conditions of an engine. The optical access is provided via quartz windows. The design of the chamber facilitates investigations concerning the influence of different liners and position of the igniter on the ignition process, due to the modular approach of its design. 


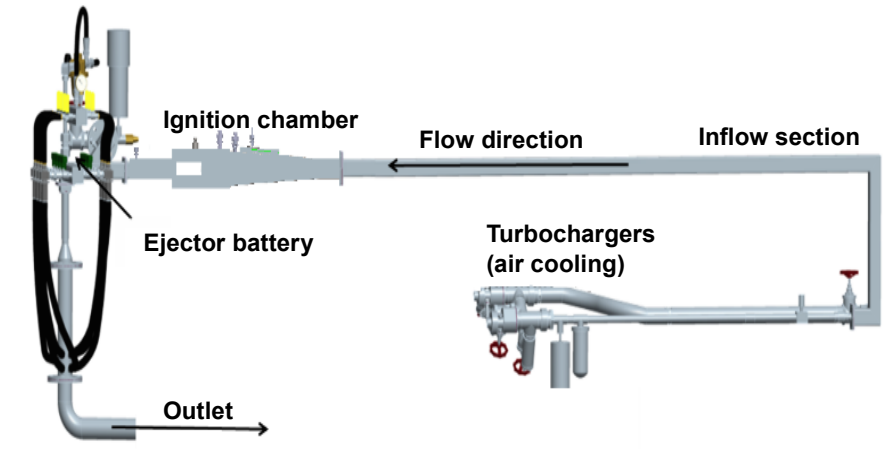

FIGURE 1: OVERVIEW OF THE ISCAR RIG

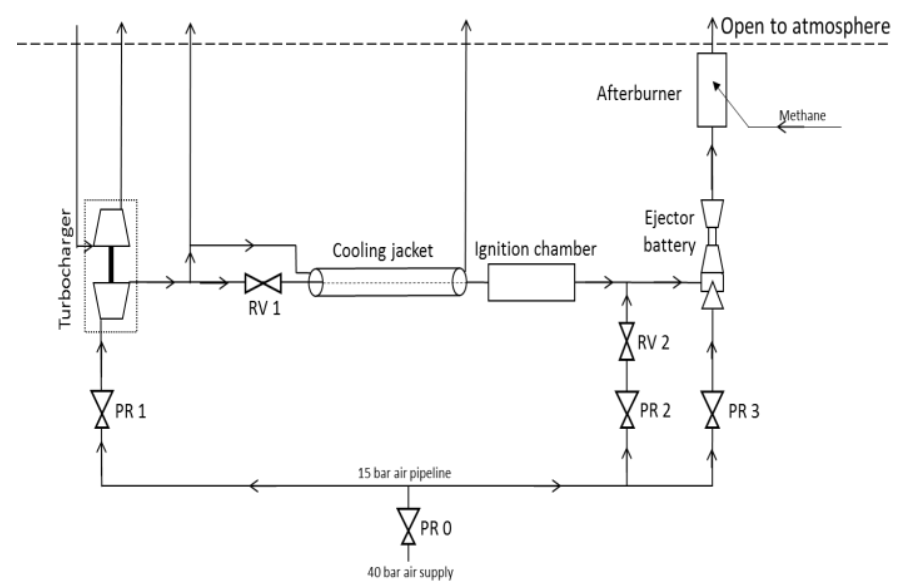

FIGURE 2: SCHEMATIC VIEW OF THE ISCAR RIG [7]

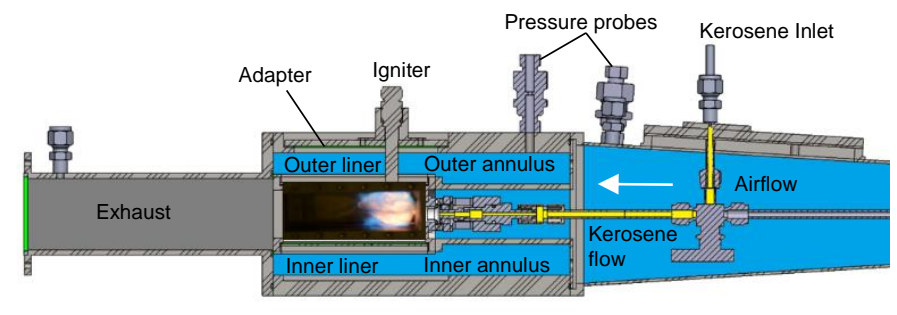

FIGURE 3: CUT VIEW OF THE IGNITION CHAMBER

The test matrix consists of 12 different operating conditions, listed in Table 1, with varied ignition chamber pressure, temperature, the pressure drop across the nozzle and fuel to air ratio (FAR).

The experimental investigations are conducted with a realistic ignition and injection systems to increase the technical relevance of the study. In Figure 4, the flow field generated by the swirler is illustrated. The swirled jet undergoes vortex breakdown due to the presence of a positive pressure gradient on the swirler axis, which leads to negative axial velocities around the centerline of the combustor, creating a strong inner recirculation zone (IRZ). In addition, the ignition device generates sparks at a rate of approximately $7 \mathrm{~Hz}$, whilst the igniter is flush mounted at the inner surface of the outer liner, as shown in Figure 3.

\begin{tabular}{c|c|c|c}
$\begin{array}{c}\mathbf{P}_{\mathbf{3}} \\
{[\mathbf{b a r}]}\end{array}$ & $\begin{array}{c}\mathbf{T}_{\mathbf{3}} / \mathbf{T}_{\text {ref }} \\
{[-]}\end{array}$ & $\begin{array}{c}\mathbf{F A R} / \mathbf{F A R} \mathbf{R}_{\text {ref }} \\
{[-]}\end{array}$ & $\begin{array}{c}\Delta \mathbf{p}_{\text {nozzle }} / \Delta \mathbf{p}_{\text {nozzle,ref }} \\
{[-]}\end{array}$ \\
\hline 1.00 & 1.103 & 0.681 & 0.954 \\
\hline 1.00 & 0.948 & 0.562 & 1.200 \\
\hline 0.67 & 0.948 & 0.824 & 1.252 \\
\hline 0.65 & 1.003 & 0.867 & 1.197 \\
\hline 0.65 & 0.948 & 0.890 & 1.233 \\
\hline 0.64 & 1.000 & 1.000 & 1.000 \\
\hline 0.55 & 0.948 & 0.962 & 1.357 \\
\hline 0.54 & 0.977 & 1.062 & 1.206 \\
\hline 0.54 & 0.948 & 1.024 & 1.243 \\
\hline 0.53 & 0.974 & 1.143 & 1.087 \\
\hline 0.44 & 0.948 & 1.229 & 1.316 \\
\hline 0.43 & 0.948 & 1.333 & 1.169
\end{tabular}

TABLE 1: TEST MATRIX

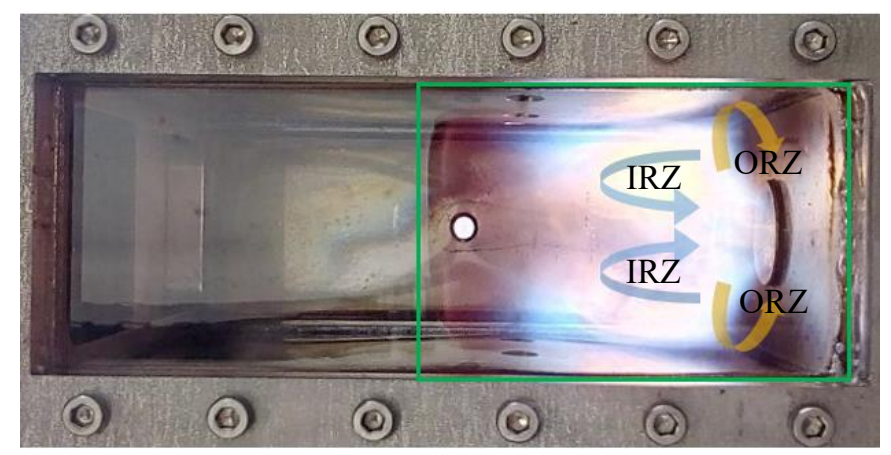

FIGURE 4: FLOW FIELD DOWNSTREAM THE INJECTOR, BOUNDARIES OF THE INTERROGATION WINDOW (GREEN RECTANGULAR SHAPE $\mathrm{H}_{0} \times \mathrm{L}_{0}$ )

The test procedure consists of three distinctive phases. Preparation:

- Set air mass flux, temperature, and pressure

- Reach steady-state conditions

Light on:

- Start and stabilize the fuel flow

- Start the igniter

- Light on event (producing spark for $10 \mathrm{sec}$ )

- Successful or unsuccessful event

Shut off:

- Turn off the igniter

- Purging of the remaining kerosene from the pipe

\section{RESULTS AND DISCUSSION}

The present study aims to provide insights concerning the ignition capability under high altitude conditions for two different configurations (liners without and with effusion cooling). 
Only one experiment for each operating condition is considered inadequate to create the ignition probability map. Taking into account statistics and the time needed for each experiment to be completed, four experiments for each operating point of the test matrix were performed.

\subsection{High altitude relight experiment without effusion cooling}

The high altitude relight capability of this investigation was carried out initially with a constant fuel flow, the same for each operating condition of the test matrix.

In Figure 5, the height of each bar illustrates the ignition probability with respect to pressure and normalized temperature. An increase in the simulated altitude influences the probability of a successful ignition event. Low pressure and temperature conditions hamper several important mechanisms governing the relight process. Probably the most prominent among them is the fuel's atomization and chemical reaction rate, which are both detrimentally affected by high altitude conditions. The general trend indicates reduction of the ignition probability as the conditions in the chamber become more adverse. It seems that the effect of pressure is more obvious due to the limited substantial relative variations obtainable within the practical temperature range of a relight event. More specifically, for the first two points in Figure 5, the pressure in the ignition chamber is atmospheric and the measured ignition probability is $100 \%$ for both operating conditions, despite the relative temperature reduction of $14 \%$. In addition, in the lower pressure range, the ignition probability is decreasing from $100 \%$ at 1 bar (4 out of 4 ) to $50 \%$ at $0.55-0.53$ bar. Due to the transition to the lowest pressure range at $0.44-0.43$ bar, the decrease of approximately $20 \%$ in pressure leads to a further $25 \%$ reduction of the ignition probability. In spite of the limited relative variations, the temperature's effect is apparent within the moderate pressure range of $0.67-0.64$ bar. It is evident that a temperature reduction of approximately $5 \%$ decreases the ignition probability $25 \%$.

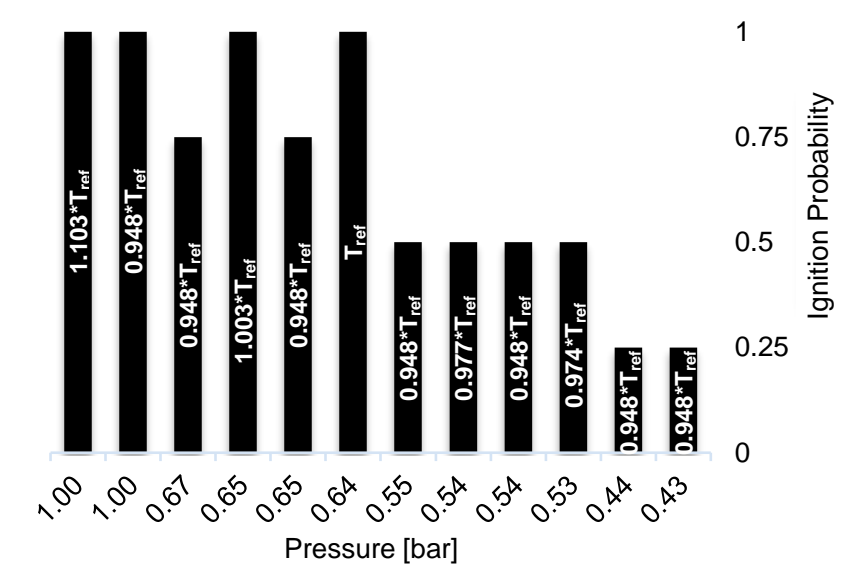

FIGURE 5: IGNITION PROBABILITY WITH A CONSTANT FUEL MASS FLOW WITH RESPECT TO PRESSURE AND NORMALIZED TEMPERATURE

As mentioned afore, the high altitude relight experiments were performed with a constant fuel mass flow. It is also quite important to investigate the effect of pressure and temperature on the minimum FAR for a potential ignition. Based on the constant fuel mass flow of the prior investigation, the fuel quantity was subsequently reduced gradually with a defined step for each operating condition. The minimum FAR was calculated using the amount of fuel of the step where at least one successful event out of four ( $25 \%$ ignition probability) was detected. The general trend indicates an increase of minimum FAR with increased altitude, as shown in Figure 6. The trend of the minimum FAR confirms earlier studies $[8,9]$ which indicated the same behavior. There is a sequence of events, which explains the latter behavior. The airblast atomizer performs poorly under lowpressure conditions due to the low air density. This results in large droplets, which in combination with low temperature reduces significantly the evaporation rate leading to leaner mixtures due to the variation of the local FAR [10] in the region of the spark. This is overcome by creating richer mixtures with increased altitude.

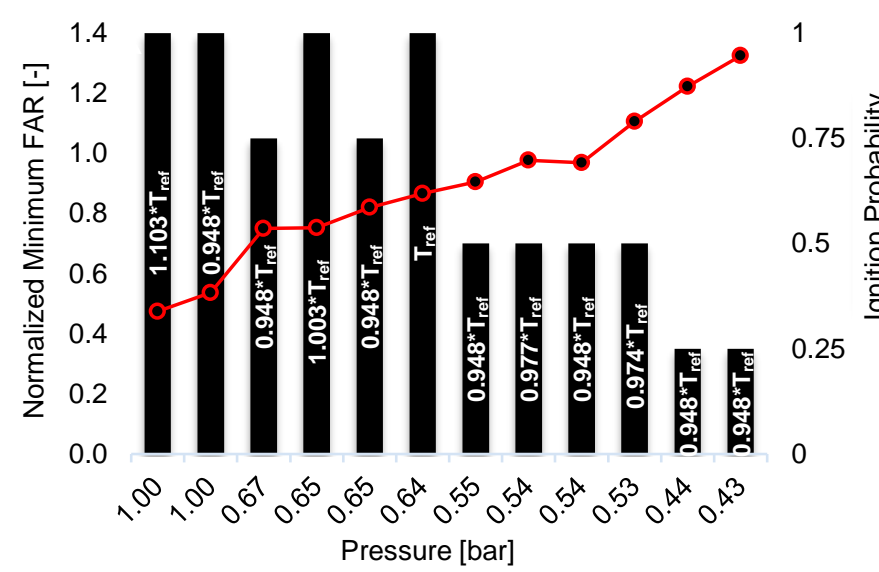

FIGURE 6: NORMALIZED MINIMUM FAR (RED CURVE) WITH RESPECT TO PRESSURE AND NORMALIZED TEMPERATURE

\subsubsection{Ignition timing}

A Si-photodiode (Thorlabs PDA36A-EC) was used to detect the time interval between the first spark and the onset of the flame for each operating condition. In addition, with this measurement technique, the number of sparks needed for a successful ignition event can be measured, as it is shown in Figures 7-9. Each equally distributed peak represents an individual spark.

As stated above, ignition probability decreases as long as the conditions in the chamber become more adverse. This implies that in the first 10 seconds less successful ignition events were captured, indicating that ignition timing increases on average, with increased altitude. However, ignition has a rather stochastic nature and depends strongly on the probability of finding a flammable mixture in the region of the spark. As it is shown in Figures 8 and 9, the ignition timing of a successful ignition event for the reference operating point, was longer than the one of the 0.44 bar, albeit this was the only successful event for the latter condition within the first 10 seconds. 


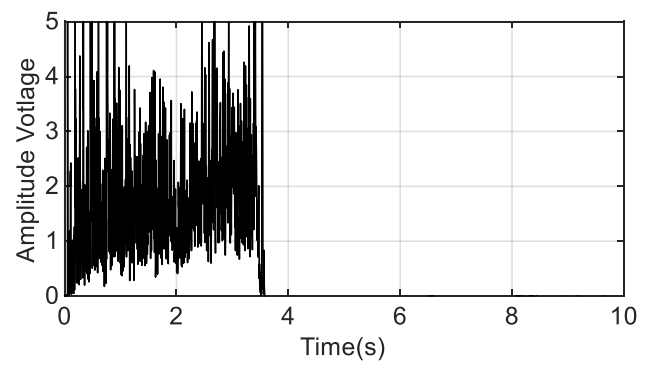

FIGURE 7: IGNITION TIMING AT 1 BAR AND $1.103 * \mathrm{~T}_{\text {ref }}$

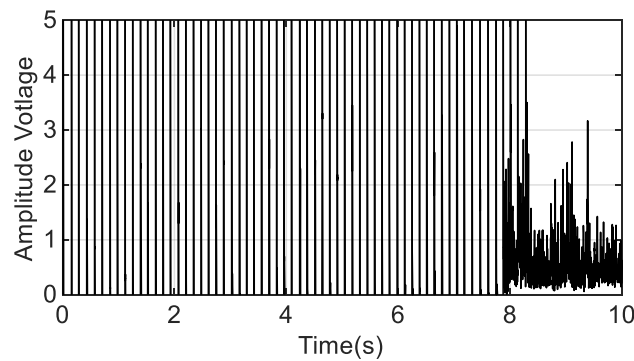

FIGURE 8: IGNITION TIMING AT 0.64 BAR AND $\mathrm{T}_{\text {ref }}$

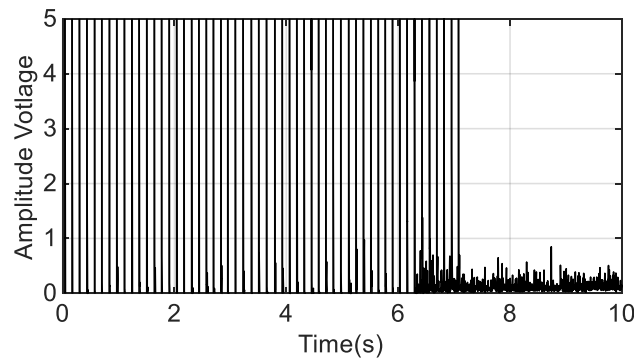

FIGURE 9: IGNITION TIMING AT 0.44 BAR AND $0.948^{*} \mathrm{~T}_{\text {ref }}$

\subsubsection{High-Speed Recording}

A high-speed video camera was used to record the unsteady flame kernel generation and propagation. The camera (LaVision HighSpeedStar 5) was arranged perpendicular to the flow direction, providing flame luminosity images at a repetition rate of $1500 \mathrm{~Hz}$. In figure 10, a successful ignition event for the reference operating point of the test matrix is illustrated.

The progress of the ignition process can be summarized as follows:

- The first frame of the recording at $0 \mathrm{~ms}$ is showing a bright kernel which is produced by the plasma emission and propagates upstream towards the nozzle.

- The light emission from the flame kernel decays rapidly, and a weak emission from the kernel persists into the next two frames up to $20 \mathrm{~ms}$.

- In the following $15 \mathrm{~ms}$, a dark period appears which shows no visible evidence. This ignition delay may contain the droplet evaporation process or a shift in the emission wavelength beyond the detection range.

- At $50 \mathrm{~ms}$, the flame radiation reappears.
- At $68 \mathrm{~ms}$ it moves in the inner recirculation (IRZ) zone.

- Then the flame volume increases and covers the lower part of the chamber at $78 \mathrm{~ms}$.

- Due to the further increase of the flame volume a stable burning configuration is observed at $159 \mathrm{~ms}$.

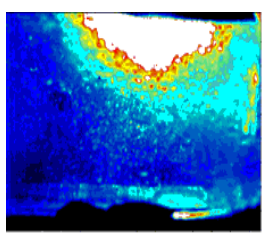

$0 \mathrm{~ms}$

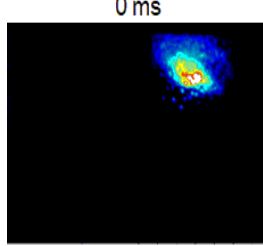

$10 \mathrm{~ms}$

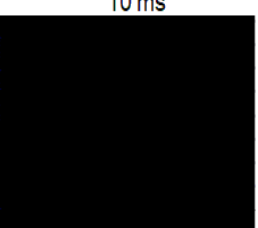

$40 \mathrm{~ms}$

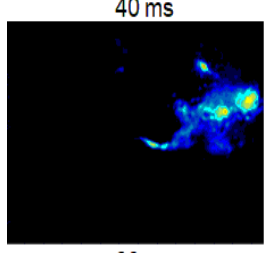

$68 \mathrm{~ms}$

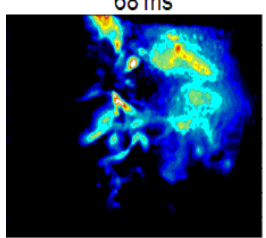

$130 \mathrm{~ms}$

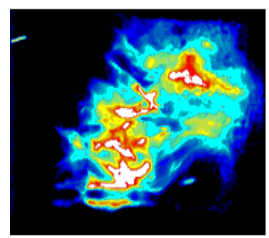

$180 \mathrm{~ms}$

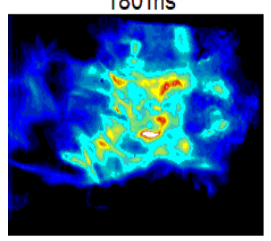

$283 \mathrm{~ms}$

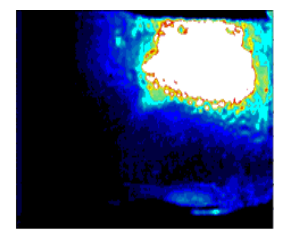

$2 \mathrm{~ms}$

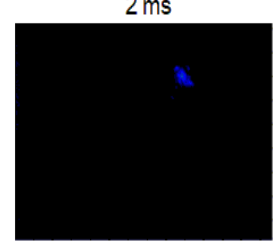

$20 \mathrm{~ms}$

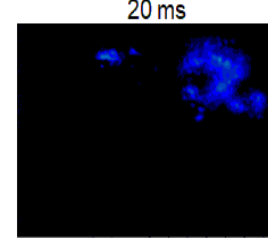

$50 \mathrm{~ms}$

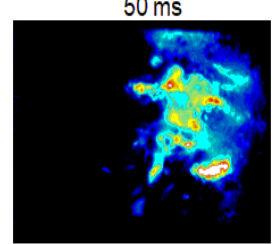

$78 \mathrm{~ms}$

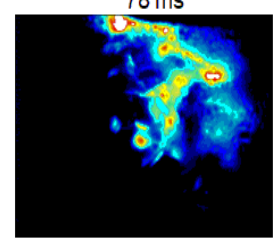

$150 \mathrm{~ms}$

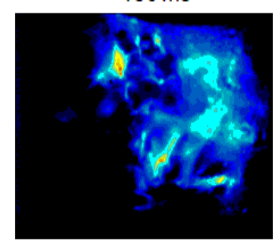

$200 \mathrm{~ms}$

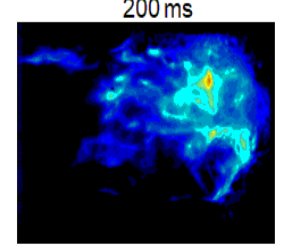

$370 \mathrm{~ms}$

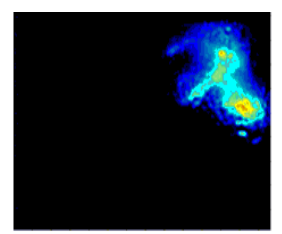

$5 \mathrm{~ms}$

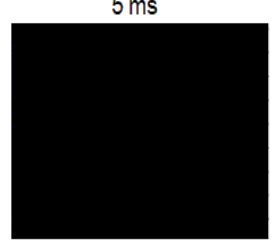

$25 \mathrm{~ms}$

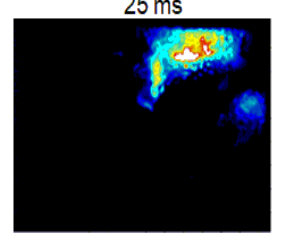

$52 \mathrm{~ms}$

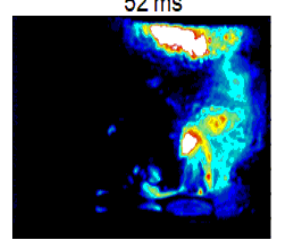

$100 \mathrm{~ms}$

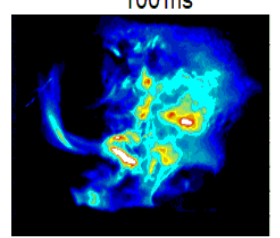

$159 \mathrm{~ms}$

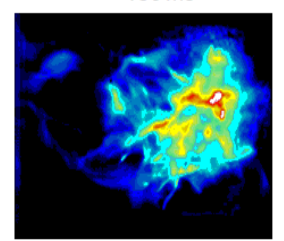

$221 \mathrm{~ms}$

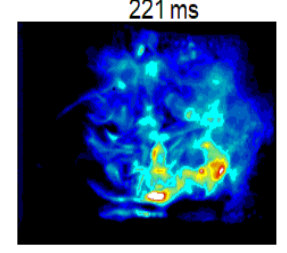

$450 \mathrm{~ms}$
FIGURE 10: IGNITION SEQUENCE, REFERENCE OPERATING POINT AT, FLOW FROM RIGHT TO LEFT 


\subsection{High altitude relight experiment with effusion cooling}

In the second configuration, effusion cooling is introduced. The comparability between both configurations was achieved by maintaining the same pressure drop across the nozzle and the liners, following the same experimental procedure, conducting experiments for the same simulated altitude with or without effusion cooling.

Detailed effective area measurements of the second configuration indicated a ratio of air mass flow between the experiment with and without effusion cooling, $\frac{\dot{m}_{a c t}}{\dot{m}_{a t}}=1.6$. In Figure 11, the high altitude relight capability at fixed fuel mass flow with effusion cooling is illustrated. A different behavior of the ignition probability compared to the first configuration is evident. It seems that the additional cooling air flowing parallel to the liners creates favorable ignition conditions. This resulted in a probability of $100 \%$ (4 out of 4 ) for a successful ignition event for each operating condition of the test matrix.

In addition, Figure 11 depicts the minimum FAR based on the injector for $100 \%$ ignition probability. The different approach in determining the minimum FAR between the two configurations (from $25 \%$ to $100 \%$ ) was within the scope of increasing the technical relevance of the study, whereby the minimum FAR was determined following the same approach as on a real engine. As mentioned afore, with or without cooling interaction the poor performance of the airblast atomizer with increased altitude is compensated by creating richer mixtures in the primary zone.

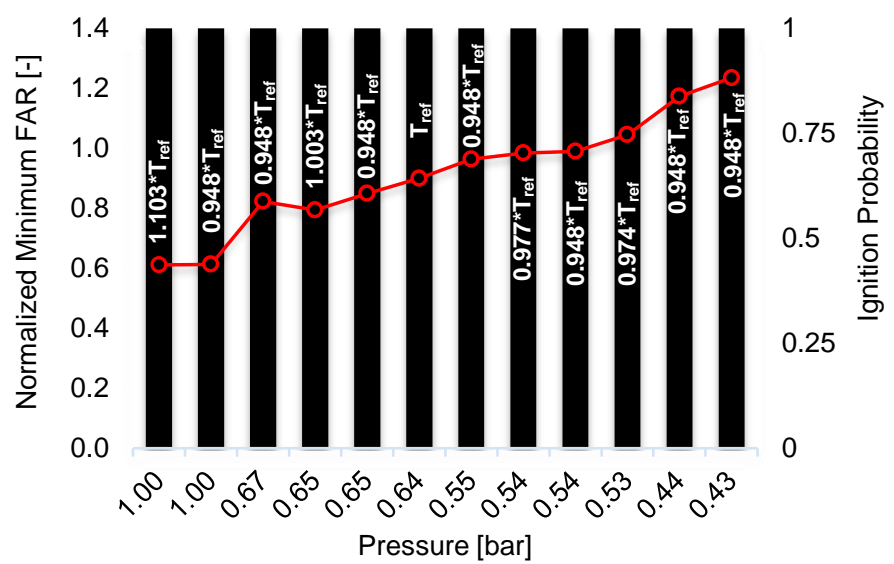

FIGURE 11: IGNITION PROBABILITY WITH CONSTANT FUEL MASS FLOW WITH RESPECT TO PRESSURE AND NORMALIZED TEMPERATURE - MINIMUM FAR DETERMINATION (RED CURVE)

In order to achieve a direct comparison between the two configurations, measurements of the minimum FAR for $100 \%$ probability have been also carried out without effusion cooling. In Figure 12, the normalized minimum FAR comparison with or without cooling interaction is illustrated. It is clear that even though the two configurations show identical trends, with effusion cooling the minimum FAR is lower, confirming that the additional air in the region of the spark supports an easier ignition process.
The four operating conditions, shown in Figure 12, consisting of two pairs of pressure and temperature ranges. It is evident that even for points with almost the same altitude there is a significant increase in the minimum FAR. The normalized predicted SMD has been calculated by the empirical correlation (Eq. (1)) proposed by El-Shanawany \& Lefebvre [11]. It consists of two separate terms, one of which is dominated by parameters of the Weber number (i.e. surface tension, density, relative velocity) and the other by the Ohnesorge-number (i.e. dynamic viscosity). For the points in Figure 12 with similar pressure and temperature, the change in SMD can explain the significant increase of the minimum FAR.

$$
\begin{gathered}
\frac{S M D}{D_{h}}=\left(1+\frac{1}{A L R}\right)\left[0.33\left(\frac{\sigma_{l}}{\rho_{a} U_{a}{ }^{2} D_{P}}\right)^{0.6}\left(\frac{\rho_{l}}{\rho_{a}}\right)^{0.1}\right. \\
\left.+0.068\left(\frac{\mu_{l}{ }^{2}}{\sigma \rho_{l} D_{p}}\right)^{0.5}\right]
\end{gathered}
$$

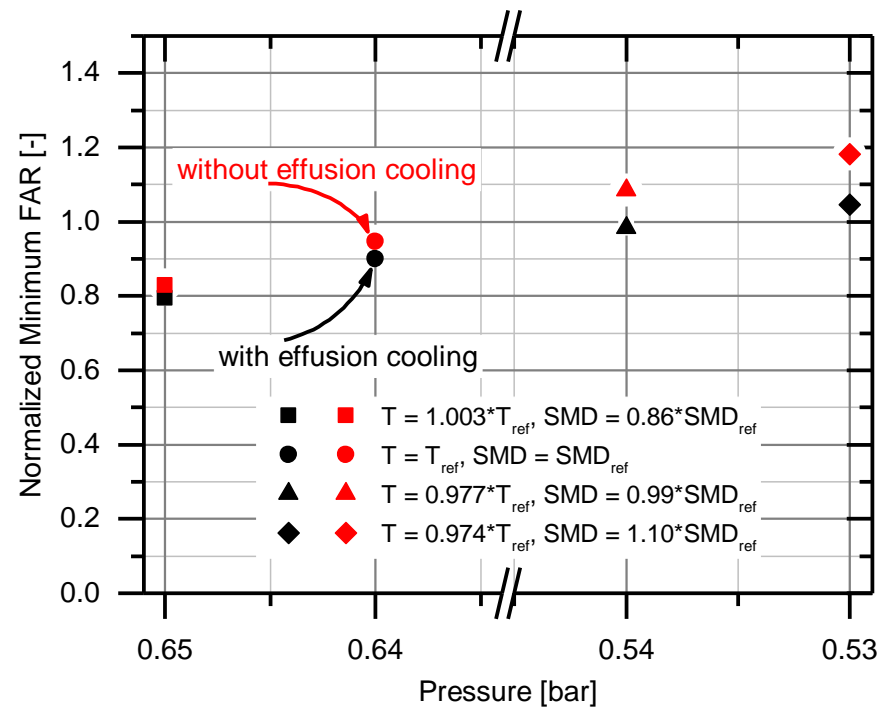

FIGURE 12: DIRECT COMPARISON OF THE TWO CONFIGURATIONS WITH RESPECT TO MINIMUM FAR

Another aspect, which has to be taken into account for the interpretation of the minimum FAR behavior, is the effect of Markstein number on the laminar burning velocity. Measurements have shown that the Markstein number of kerosene Jet A-1 is negative for rich mixtures under the investigated conditions [12]. In this case, flame stretch (caused by turbulence) will have a positive effect on the laminar burning velocity towards richer fuel/air mixtures.

\subsubsection{Ignition timing with effusion cooling}

In Figures 13-15, the ignition timing is illustrated. It is obvious that on average, the time interval between the first spark and the onset of the flame increases with increased altitude, following the same behavior compared to the results of the first configuration. 
The additional air creates favorable conditions for ignition, which leads to a significant reduction of the ignition timing compared to the previous configuration under the same operating conditions. The increased ignitability of the second configuration is expressed by the higher ignition probability within 10 seconds detected throughout the conduct of the second experimental campaign.

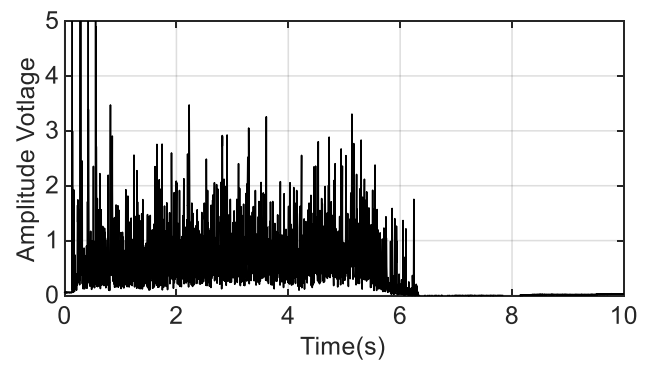

FIGURE 13: IGNITION TIMING AT 1 BAR AND $1.103 * \mathrm{~T}_{\text {ref }}$

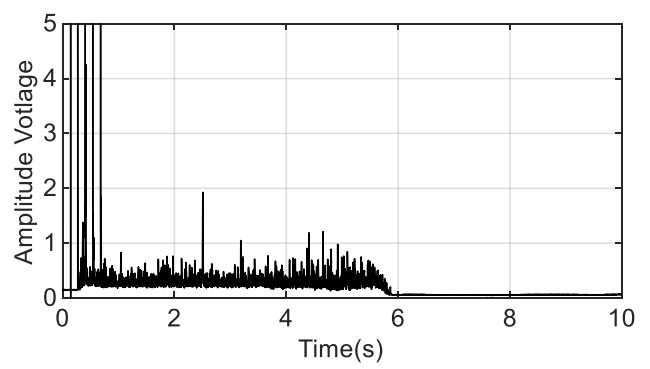

FIGURE 14: IGNITION TIMING AT 0.64 BAR AND $\mathrm{T}_{\mathrm{ref}}$

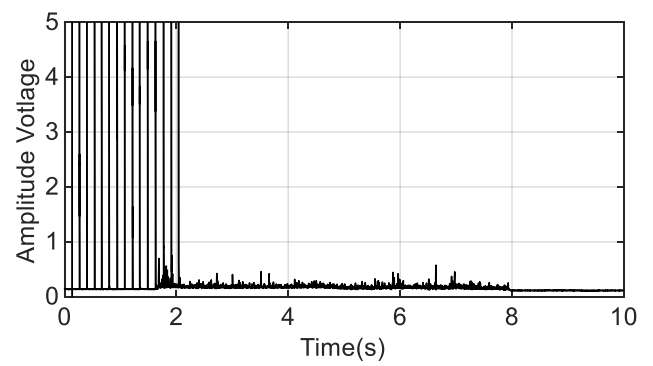

FIGURE 15: IGNITION TIMING AT 0.44 BAR AND $0.948^{*} \mathrm{~T}_{\mathrm{ref}}$

\subsection{Development of an image processing code for the evaluation of the ignition process}

An image processing code was developed to extract further information from the high-speed recordings in a systematic way. A simplified block diagram of the code is shown in Figure 16. As mentioned afore, the evolution of the flame during the ignition attempts was recorded with a high-speed camera and stored as a grayscale image, as shown in Figure 16(a). In order to highlight the details and the boundary of the flame, the original image was filtered with Gaussian kernel and intensity enhancement (Figure 16(b)). Moreover (Figure 16(c)), the image was segmented using the method of maximum intercluster variance, a self-adaptive threshold determination method (known as Otsu's method) [13]. After applying a boundary trace algorithm using Matlab (see Figure 16(d)), quantitative spatial information of the flame such as tracking of flame's luminosity center, height, and length of the flame and growth rate were derived. In addition, the behavior of the ignition sequences for the same or different operating conditions was evaluated and overall trends and characteristic behaviors were identified.

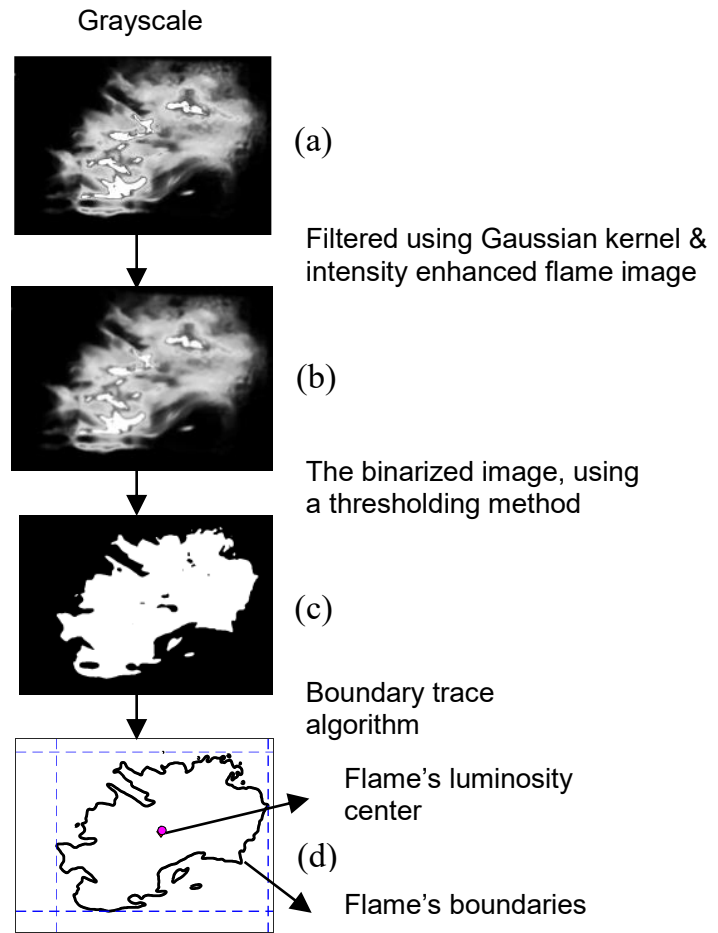

FIGURE 16: BLOCK DIAGRAM OF THE IMAGE PROCESSING CODE

\subsubsection{Preliminary observations-total intensity variation}

The total intensity variation measured by the camera in each high-speed recording provides a simple appraisal of the combustion activity during an experimental trial. A preliminary analysis used these data to characterize the influence of the altitude on the timescales of ignition success and captured the behavior of the stabilized flame. In Figures $17 \mathrm{a} \& 17 \mathrm{~b}$, the recovery times for the individual ignition trials of the sea level and high altitude respectively, are illustrated. The high-intensity initial signal due to the spark decays to a low level within approximately $10 \mathrm{~ms}$, approaching the black period, which was also detected in the previous configuration (see Figure 10). Subsequently, it grows and develops by emitting low intensity, following the same behavior, which was captured in the previous configuration by flame visualization (Figure 10). The recovery time is the period following the spark when the signal rises for the first time to exceed a threshold value of $2 \times 10^{4}$ counts (see orange line in Figure 17a \& 17b). A typical behavior of an unsuccessful ignition event is shown in Figure 17c. The intensity count never exceeds the threshold value during a failure, but it always recovers considerably after having exceeded it during a successful event. By taking into account the individual ignition trial for each operating condition, it is clear that the recovery time 
increases with increased altitude. Furthermore, in Figures 18a \& $18 \mathrm{~b}$, the mean flame recovery and the subsequent flame progress out of four ignition trials together with the standard deviation is illustrated. It is conspicuous that in the early stages flame kernel generation and propagation is very stochastic. Due to this randomness, performing four experiments per operating point provides an indication, but it is not considered an adequate amount of data to correlate the flame's recovery time with the global operating conditions.

Moreover, visual examination of the high-speed images acquired following flame recovery, suggests that the stabilized flame fluctuates approximately sinusoidal.
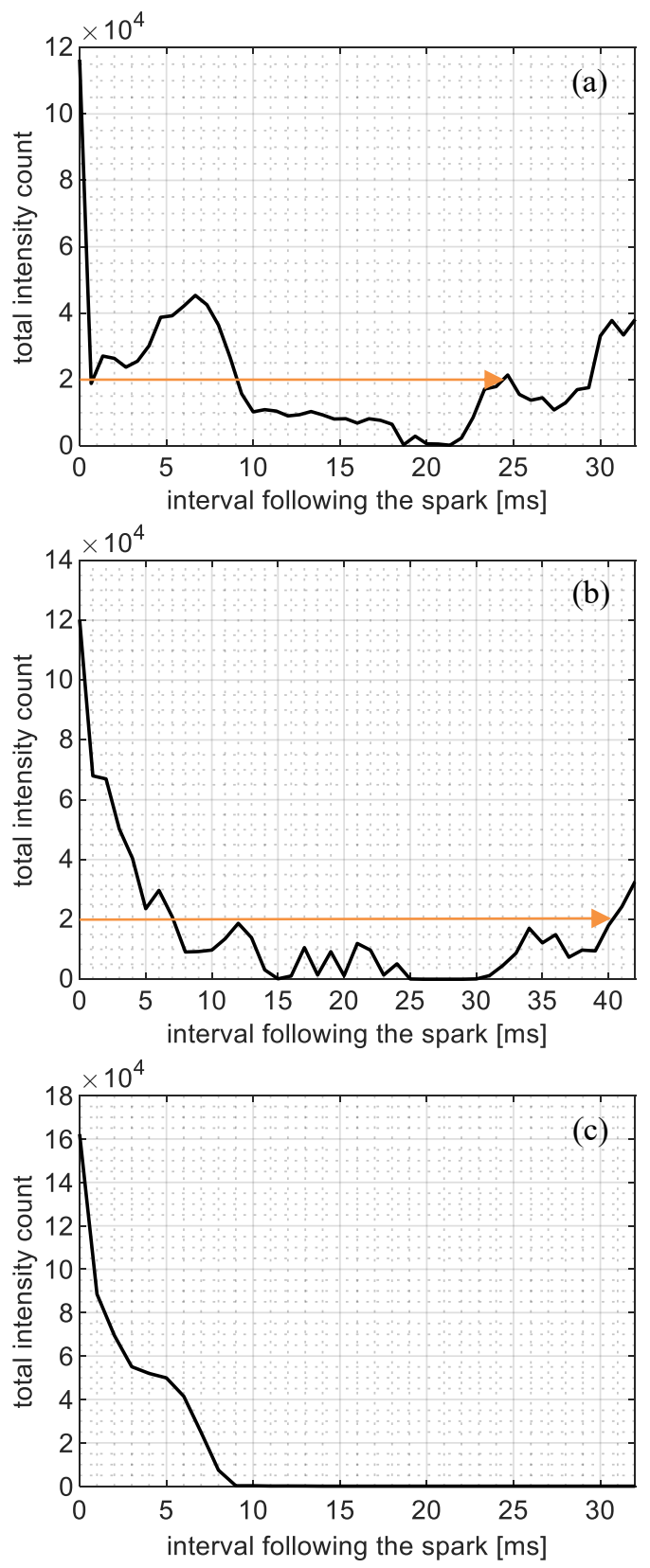

FIGURE 17: FLAME RECOVERY - (a) SEA LEVEL - (b) 0.44 BAR $0.948^{*} \mathrm{~T}_{\text {ref }}-$ (c) UNSUCCESSFUL IGNITION EVENT
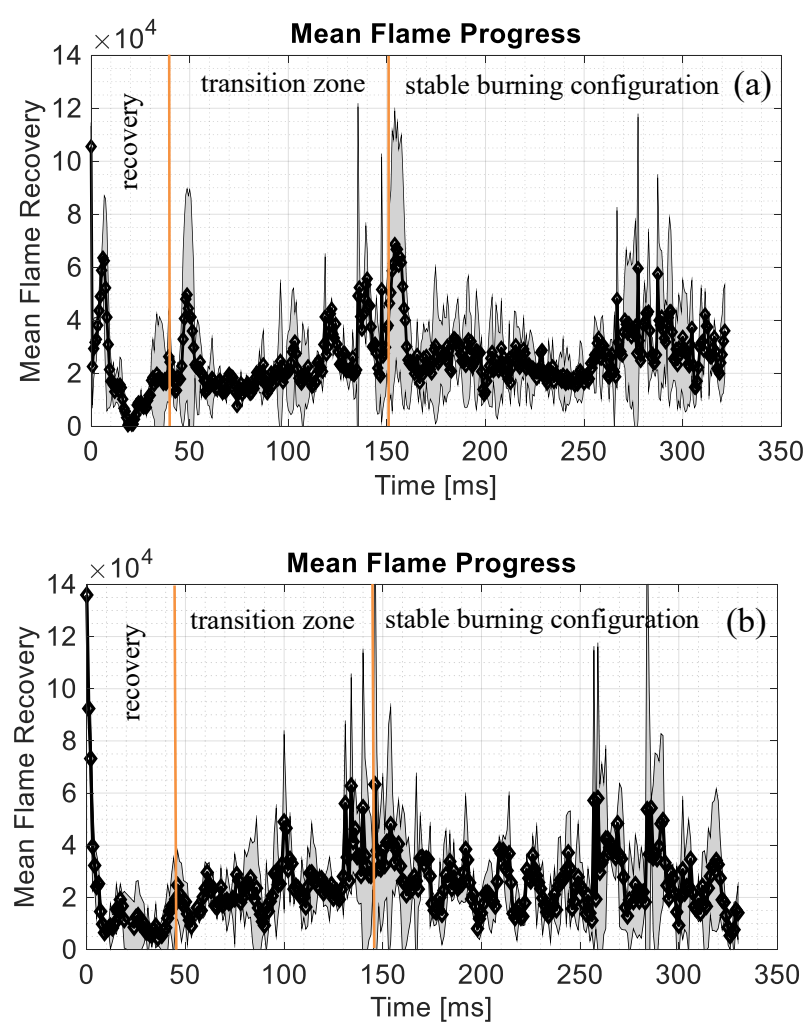

FIGURE 18: MEAN FLAME PROGRESS - (a) SEA LEVEL - (b) 0.44 BAR $0.948 * \mathrm{~T}_{\text {ref }}$

\subsubsection{Flame Trajectories}

Trajectory plots of the flame's motion have been examined to identify the behavior of the ignition development. The flame tracking algorithm was used to generate a flame trajectory plot for each high-speed recording of an ignition attempt. The luminosity center was extracted via the gray image using the weighting method as follows:

$$
\begin{aligned}
& x=\frac{\sum_{i=1}^{N} x_{i} I_{\left(x_{i}, y_{i}\right)}}{\sum_{i=1}^{N} I_{\left(x_{i}, y_{i}\right)}} \\
& y=\frac{\sum_{i=1}^{N} y_{i} I_{\left(x_{i}, y_{i}\right)}}{\sum_{i=1}^{N} I_{\left(x_{i}, y_{i}\right)}}
\end{aligned}
$$

Where $x$ and $y$ are the resulting horizontal and vertical coordinates of mass center; $i$ is the index of each pixel of the $N$ total number of pixels within the flame region; $x_{i}$ and $y_{i}$ are the horizontal and vertical coordinates of each pixel within the flame region; $I_{\left(x_{i}, y_{i}\right)}$ is the luminosity corresponding to the coordinates of $x_{i}$ and $y_{i}$ extracted by the gray-scale image.

In Figure 19, the mean (out of four experiments) movement of the flame's luminosity center in different periods, for the reference point, is illustrated. The boundaries of the graphs represent the interrogation window of the high-speed camera. The initial activity below the igniter depicted by the star is shown in Figure 19(a). In Figures 19(b)-(f) the movement of the flame's luminosity center following the initial activity is plotted. For 
each subsequent individual movement, the color of the curve is red, whereas the black color represents the movement already established in previous time scales. The flame kernel travels upstream, before developing strongly in the upper upstream quadrant of the combustion chamber. When the recovery occurs after the "black" period, the flame propagates in the inner recirculation zone, confirming the behavior captured by flame visualization of the first configuration (see Figure 11). Finally, in Figure 20 the flame trajectory map of the whole recording is plotted. The plot is color-coded with respect to time, starting from dark blue to dark red for $0 \mathrm{~ms}$ and $500 \mathrm{~ms}$ respectively. It is clear that after the movement in the inner recirculation zone, the flame has spread and occupies a significant proportion of the interrogation window. Approximately at $150 \mathrm{~ms}$ (light blue), the flame already reached a stable burning condition. The flame trajectory for both configurations is similar while the time scale of the flame's evolution is shorter in the configuration with effusion cooling due to the reduced residence time resulting from the higher air mass flow.
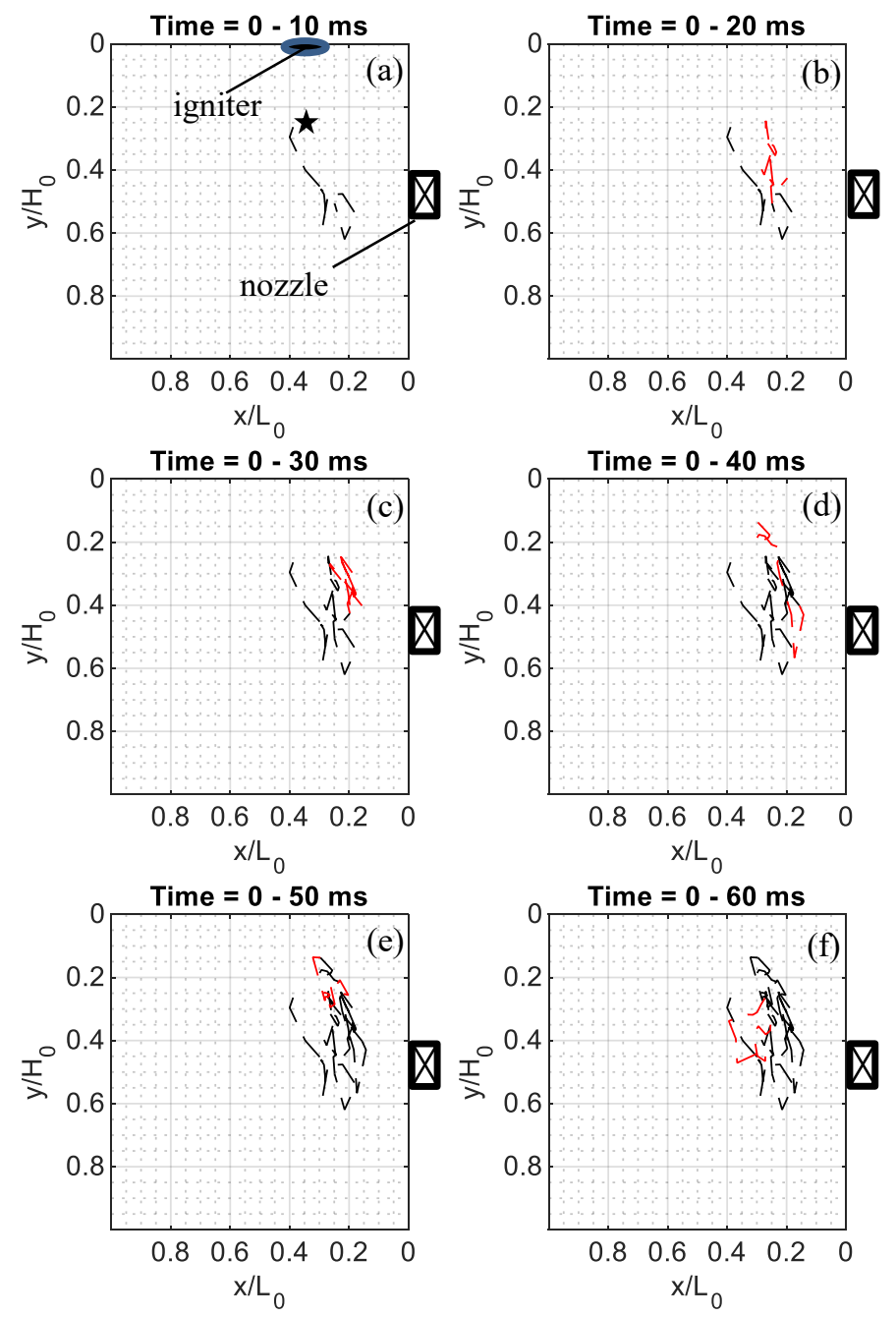

FIGURE 19: TRACKING OF THE FLAME'S LUMINOSITY CENTER, FLOW DIRECTION FROM RIGHT TO LEFT

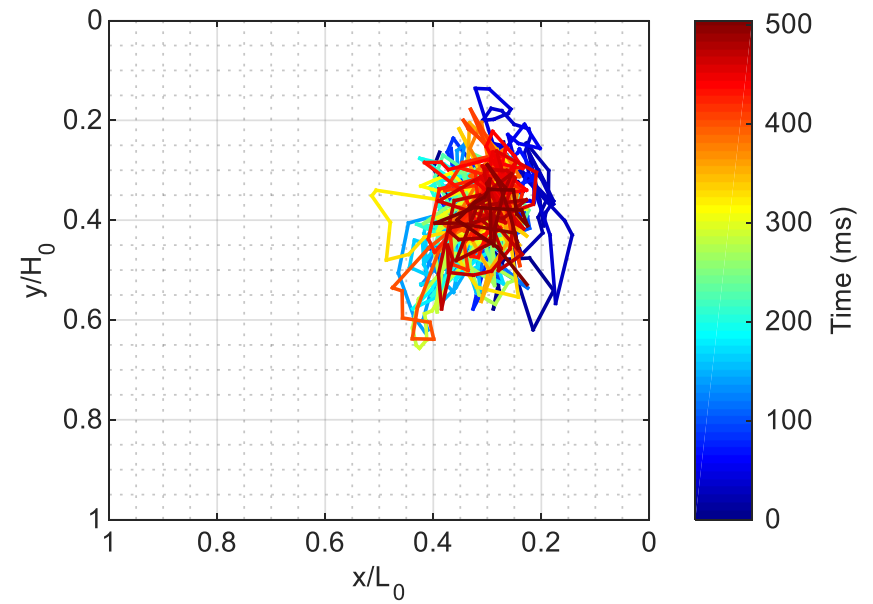

FIGURE 20: COLOUR-CODED MOVEMENT OF FLAME'S LUMINOSITY CENTER, FLOW DIRECTION FROM RIGHT TO LEFT

\section{CONCLUSION}

An investigation on the ignition of flowing kerosene (Jet A1)-air mixtures under altitude relight conditions was carried out. Results concerning the influence of thermodynamic conditions (pressure and temperature) on the ignition capability for two different liner configurations (without and with effusion cooling), were obtained.

In the configuration without effusion cooling, the pressure seems to dominate the probability of having a successful ignition event for the specified maximum time of spark production, due to the limited substantial variations of temperature in the investigated test matrix. The general trend indicates that an increase in the simulated altitude results in a decrease in the ignition probability. The configuration with effusion cooling was not so sensitive to pressure and temperature variations in terms of ignition probability. The additional air injected in the primary zone via effusion cooling holes seems to create favorable conditions for the ignition process, especially at increased simulated altitude compared to the first configuration, resulting in a $100 \%$ probability for a successful event for each operating condition of the test matrix.

In addition, the minimum FAR for a successful ignition event was determined. In both configurations, with increased simulated altitude the minimum FAR was also increased. Low temperature and pressure create adverse conditions for the atomized fuel, which seems to be overcome by creating richer fuel to air ratios in the primary zone. Among the operating conditions with almost the same simulated altitude, the ones with higher predicted SMD experienced also an increase in the minimum FAR. In order to verify the SMD contribution to the minimum FAR prediction, further investigations are necessary. This includes spray measurements under high altitude conditions with laser optical techniques, in order to enhance the knowledge concerning dominant parameters governing the ignition process.

The effect of Markstein number on the laminar burning velocity and consequently the minimum FAR behavior for 
different operating conditions should be investigated in the future.

A direct comparison of the two configurations indicated a lower minimum FAR for the configuration with effusion cooling, confirming that the additional air injected in the region of the spark aids in the ignition evolution.

Furthermore, measurements regarding the time interval between the first spark and the onset of the flame were carried out. On average, for both configurations, an increase in the simulated altitude increased in the ignition timing. Randomness was apparent in both configurations and there was no clear trend among the successful ignition events of the same operating conditions. The comparison of both configurations indicated that the ignition timing was significantly faster with effusion cooling, which confirmed the higher probability of successful ignition detection within 10 seconds.

Finally, an in-house image processing code was developed, in order to extract information systematically from the analysis of the high-speed recordings. Qualitative analysis showed that the movement of the reacting gas from the region of the spark to the inner recirculation zone is of importance for the stabilization of the flame. Additionally, quantitative spatial information of the flame's movement was derived. It was evident that the flame's luminosity center follows a similar pathway for both configurations, whilst with effusion cooling the time scale of the flame's evolution is shorter, due to the shorter flow residence time in this case.

\section{ACKNOWLEDGMENTS}

The results were obtained in the framework of the European research project SOPRANO (Soot Processes and Radiation in Aeronautical Innovative Combustors). This project has received funding from the European Union's Horizon 2020 research and innovation programme under grant agreement No 690724.

\section{REFERENCES}

[1] Agency E. A. S., 2010. "Certification Specifications for Engines CS-E”. (Amendment 3), pp. 1-166.

[2] Aggarwal S. K., 1998. "A review of spray ignition phenomena: Present status and future research". Progress in Energy and Combustion Science, 24(6), pp. 565-600.

[3] Mosbach T., Sadanandan R., Meier W., and Eggels R., 2010. "Experimental Analysis of Altitude Relight Under Realistic Conditions Using Laser and High-Speed Video Techniques". Volume 2: Combustion, Fuels and Emissions, Parts A and B.

[4] Read R. W., 2008. "Experimental Investigations into HighAltitude Relight of a Gas Turbine." Ph.D. thesis, University of Cambridge

[5] Denton M. J., Tambe S. B., and Jeng S. M., 2018. "Experimental investigation into the high altitude relight of a three-cup combustor sector". Proceedings of ASME Turbo Expo 2018, GT2018-77146.

[6] Neophytou A., Richardson E. S., and Mastorakos E., 2012. "Spark ignition of turbulent recirculating nonpremixed gas and spray flames: A model for predicting ignition probability". Combustion and Flame, 159(4), pp. 1503-1522.

[7] Majcherczyk M., Zarzalis N., and Turrini F., 2014. "Influence of the Turbulence Length Scale and Intensity on Spark Ignition of Kerosene Jet-A1-Air Mixtures at High Altitude Relight Conditions". Proceedings of ASME Turbo Expo 2014, GT2014-25332.

[8] Paxton B., Tambe S. B., and Jeng S.M., 2016. "Systems Design and Experimental Evaluation of a High-Altitude Relight Test Facility". Proceedings of ASME Turbo Expo 2016, GT2016-57089.

[9] Ballal D. R., and Lefebvre A. H., 1978. "Ignition of liquid fuel sprays at subatmospheric pressures". Combustion and Flame, 31, pp. 115-126.

[10] Read R. W., Rogerson J. W., and Hochgreb S., 2013. "Planar Laser-Induced Fluorescence Fuel Imaging During GasTurbine Relight”. Journal of Propulsion and Power, 29(4), pp. 961-974.

[11] El-Shanawany M. S., and Lefebvre A. H., 1980. "Airblast Atomization: Effect of Linear Scale on Mean Drop Size". Journal of Energy, 4(4), pp. 184-189.

[12] Vukadinovic V., Habisreuther P., and Zarzalis N., 2013. "Influence of pressure and temperature on laminar burning velocity and Markstein number of kerosene Jet A-1: Experimental and numerical study". Fuel, 111, pp. 401-410.

[13] Yang X., Shen X., Long J., and Chen H., 2012. "An Improved Median-based Otsu Image Thresholding Algorithm". AASRI Procedia, 3, pp. 468-473. 